\title{
Descending transtentorial herniation, a rare complication of the treatment of trapped fourth ventricle: case report
}

\author{
Paolo Frassanito, MD, ${ }^{1}$ Georgios Markogiannakis, MD, ${ }^{2}$ Rina Di Bonaventura, MD, ${ }^{1}$ \\ Luca Massimi, MD, PhD, ${ }^{1}$ Gianpiero Tamburrini, MD, ${ }^{1}$ and Massimo Caldarelli, MD1
}

1Pediatric Neurosurgery, Catholic University Medical School, Rome, Italy; and 'Department of Neurosurgery, Red Cross Hospital, Athens, Greece

\begin{abstract}
Descending transtentorial herniation (DTH) is a complication of raised pressure in the supratentorial compartment, usually resulting from mass lesion of several etiologies. The authors report an exceptional case of DTH complicating the implant of a CSF shunting device in the trapped fourth ventricle of a 17-year-old boy in whom a second CSF shunting device had been implanted for neonatal posthemorrhagic and postinfectious hydrocephalus. The insidious clinical and radiological presentation of $\mathrm{DTH}$, mimicking a malfunction of the supratentorial shunt, is documented. Ultimately, the treatment consisted of removal of the infratentorial shunt and endoscopic acqueductoplasty with stenting. The absence of supratentorial mass lesion and other described etiologies of DTH prompted the authors to speculate on the hydrodynamic pathogenesis of DTH in the present case.
\end{abstract}

http://thejns.org/doi/abs/10.3171/2015.3.PEDS14619

KEY WORDS aqueductoplasty; descending transtentorial herniation; Duret hemorrhage; endoscopy; paradoxical herniation; trapped fourth ventricle; hydrocephalus

$\mathrm{D}$ ESCENDING transtentorial herniation (DTH) consists of herniation of supratentorial structures through the tentorial hiatus into the posterior cranial fossa. This phenomenon usually results from increased pressure in the supratentorial compartment that is related to the presence of a mass lesion. ${ }^{5,11,14}$ More rarely, DTH has been described in the absence of mass lesion as a complication of rapid correction of hyponatremia, with subsequent swollen brain parenchyma, or lumbar CSF drainage in the presence of open skull. ${ }^{13,16,18}$ If the supratentorial pressure is not promptly relieved, the evolution of this condition may lead to acute sufferance (i.e., distortion and compression) of the brainstem, which is associated with a dismal prognosis. ${ }^{8,17}$
We report the management of a case of trapped fourth ventricle complicated by DTH, in the absence of increased pressure or mass lesion in the supratentorial compartment. Indeed, DTH resulted from a negative pressure gradient between the fourth ventricle and the supratentorial compartment. The pathogenesis and treatment of this extremely rare condition is thoroughly discussed.

\section{Case Report}

A 17-year-old boy in whom a ventriculoperitoneal (VP) shunt with a medium-pressure valve had been implanted for neonatal posthemorrhagic and postinfectious hydrocephalus showed compensated trapped fourth ventricle at

ABBREVIATIONS DTH = descending transtentorial herniation; VP = ventriculoperitoneal. SUBMITTED November 4, 2014. ACCEPTED March 23, 2015.

INCLUDE WHEN CITING Published online July 24, 2015; DOI: 10.3171/2015.3.PEDS14619.

DISCLOSURE The authors report no conflict of interest concerning the materials or methods used in this study or the findings specified in this paper. 
routine neuroimaging examinations. Neurological examination, which showed moderate cognitive delay and mild tetraparesis with hypertonus, had been stable throughout the previous follow-up. Recent MRI sequences showed increased size of the trapped fourth ventricle (Fig. 1A and B) associated with the onset of diplopia and dysphagia, progressively worsening in the last 3 months.

Thus, endoscopic aqueductoplasty with stenting via a suboccipital approach was performed. Radiological control examination confirmed the good result of the endoscopic procedure (Fig. 1C and D). After a transient period of good neurological condition, the patient's complaints recurred and neuroimaging control examination showed malfunctioning of the stent (Fig. 1E and F). A radioscopic injection test performed through the reservoir connected to the stent showed communication of the fourth ventricle, with the posterior portion of the third ventricle not communicating with the remaining supratentorial system. Therefore, a second VP shunt with a medium-pressure valve was placed in the fourth ventricle and the patient was discharged without neurological complaints.

Two weeks later, the patient presented with headache, dizziness, and vomiting, consistent with raised intracranial pressure. CT scans showed dilation of the supratentorial ventricular system, consistent with malfunctioning of the supratentorial VP shunt, although the size of the fourth ventricle appeared significantly reduced (Fig. $1 \mathrm{G}$ and $\mathrm{H}$ ). In spite of this radiological picture, surgical revision disclosed a correctly working supratentorial VP shunt and CSF examination ruled out any infection. However, the patient's neurological status continued to worsen, with onset of coma (Glasgow Coma Scale Score 7), miotic isocoric pupils with weak light reflex, bradycardia, and increased hypertonus, mainly in the right side. CT scans showed increased dilation of the supratentorial ventricles, with a diverticulum of the left lateral ventricle herniating through the tentorial hiatus. Thus, both shunting devices were exteriorized. Despite this maneuver and very low draining pressure of the external drainages, supratentorial ventricles remained large and the patient did not improve. Finally, an overdrainage of the fourth ventricle was hypothesized and the fourth ventricle external drainage was accordingly set at a $5-\mathrm{cm}$ higher draining pressure than the supratentorial drainage. Thereafter, patient progressively recovered and CT scans showed resolution of the DTH. Then, endoscopic aqueductoplasty with stenting via a frontal approach was performed (Fig. 2) and a single VP shunt was internalized; a third ventriculostomy was concomitantly performed.

The postoperative course was uneventful and CT scans confirmed the correctly working stent, thus balancing the pressure between supratentorial and infratentorial compartments (Fig. 1I and J). Although MRI studies performed 1 month after the occurrence of DTH showed signs consistent with previous sufferance of the brainstem (Fig. 3), the neurological status of the patient slowly recovered with rehabilitation therapy and he had returned to the preoperative neurological status at 1-year follow-up.

\section{Discussion}

Trapped fourth ventricle is a form of loculated hydro-
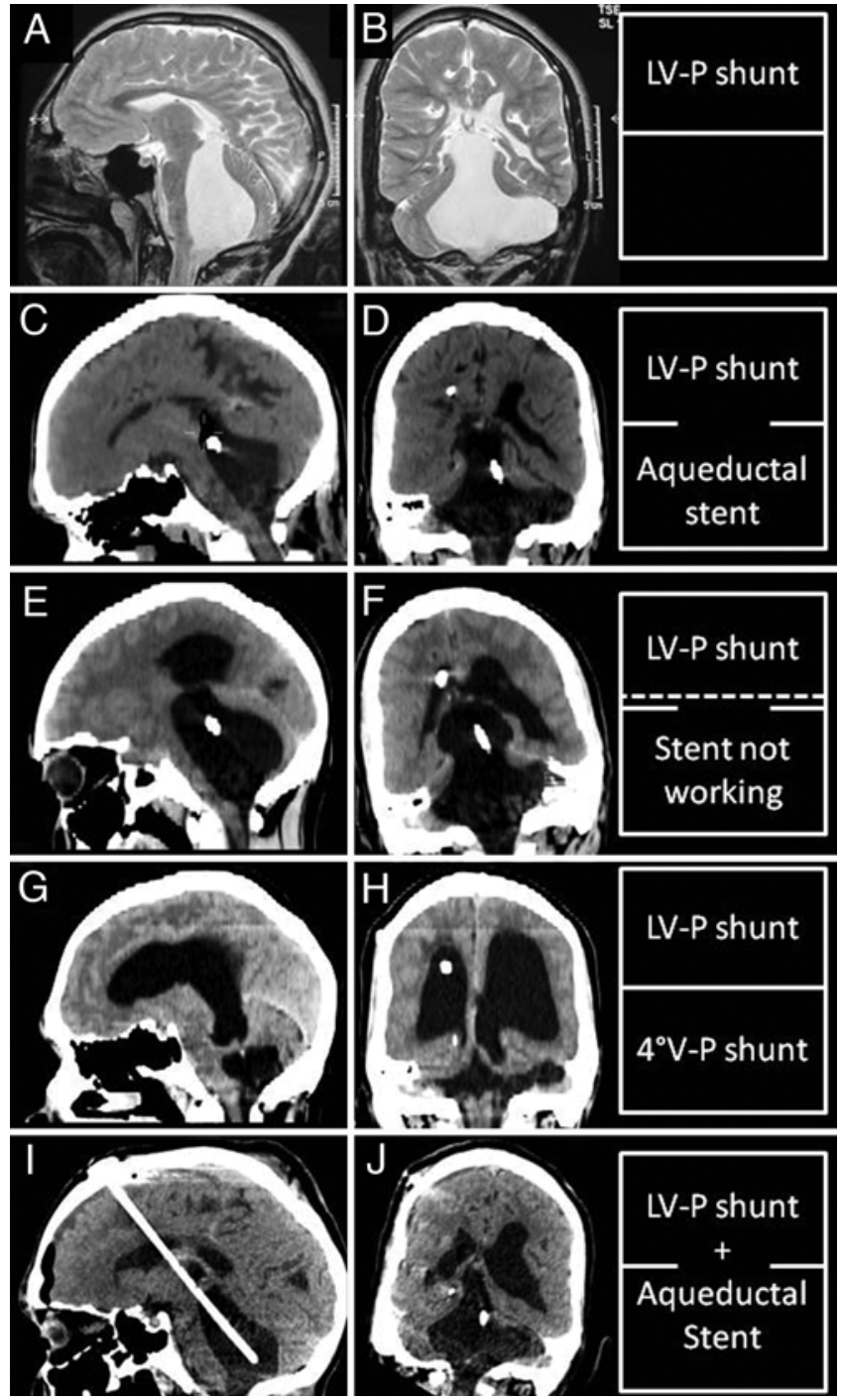

FIG. 1. Evolution of the radiological picture along with the devices implanted in the supratentorial (upper box) and infratentorial (lower box) ventricular systems, which are graphically shown on the right. LV-P = left VP; $4^{\circ} \mathrm{V}-\mathrm{P}=$ fourth ventricle-peritoneal.

cephalus in which posthemorrhagic and postinfectious origins are usually recognized. ${ }^{3,10}$ It may present with symptoms and signs of brainstem and cerebellar compression, thus requiring treatment to relieve pressure on these vital structures. However, the indications for the treatment of trapped fourth ventricle are not always so clear, because neurological symptoms may present more subtly..$^{12}$ Moreover, this condition may even be asymptomatic, therefore not requiring any treatment. In such cases, the association of marked enlargement of the fourth ventricle depicted in radiological studies with a clinical picture characterized by stable neurological parameters at presentation and at follow-up constitutes the so-called compensated trapped fourth ventricle. On these grounds, strict indications for treatment are advocated. ${ }^{28}$ In our case, the clinical and neurological status remained stable for a long time until the onset of new symptoms of oculomotor impairment and mild increase of the fourth ventricle dilation. 

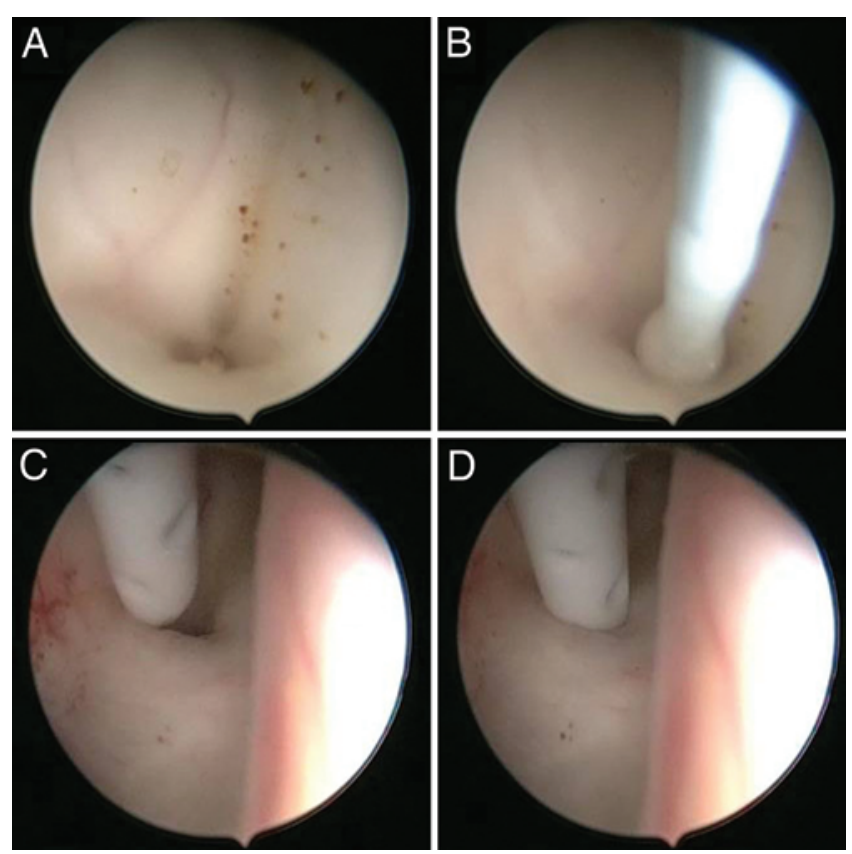

FIG. 2. Steps of the endoscopic procedure. Identification of the aqueduct (A); aqueductoplasty with Fogarty balloon (B); placement of the stent across the aqueduct (C); and confirmation of the correct positioning of the stent (D). Figure is available in color online only.

The main treatment options consist of microsurgical fenestration through a posterior fossa craniotomy/craniectomy with or without placement of a stent to the spinal subarachnoid space, to achieve a diversion of CSF, or a fourth ventricle shunt, either separate or connected via a Y-shaped connector with a supratentorial ventricular catheter. ${ }^{4,15,21,25,27}$ The advent and recent evolution of endoscopy has introduced new options in the treatment of this condition. In fact, an internal CSF diversion channel for trapped fourth ventricle may be achieved with aqueductoplasty, with or without aqueductal stent placement, or by fenestration of the superior medullary velum. ${ }^{3,67,720,22,24}$ This procedure is not devoid of complications; it carries a risk of midbrain injury, with neurological defects such as dysconjugate eye movement and Parinaud syndrome. It may be performed by approaching the aqueduct from above, when the supratentorial ventricles are dilated, or from below with a suboccipital approach, usually when a working CSF shunting device is present. ${ }^{9,19}$

In the present case, the endoscopic procedure was initially performed through a suboccipital approach because the supratentorial ventricles were slit due to the working shunt. Unfortunately this procedure did not succeed, and the stent was converted to a fourth ventricle-peritoneal shunting device, distinct from the supratentorial shunt.

The presence of 2 shunting devices, in spite of the same draining pressure of their valves, progressively led to the radiological picture of DTH of a diverticulum of the trigone in the left ventricle. This is probably due to the different design of the 2 diversion systems; the supratentorial shunt had a proximal valve, whereas the infratentorial had a distal valve that is more prone to siphoning and overdrainage.
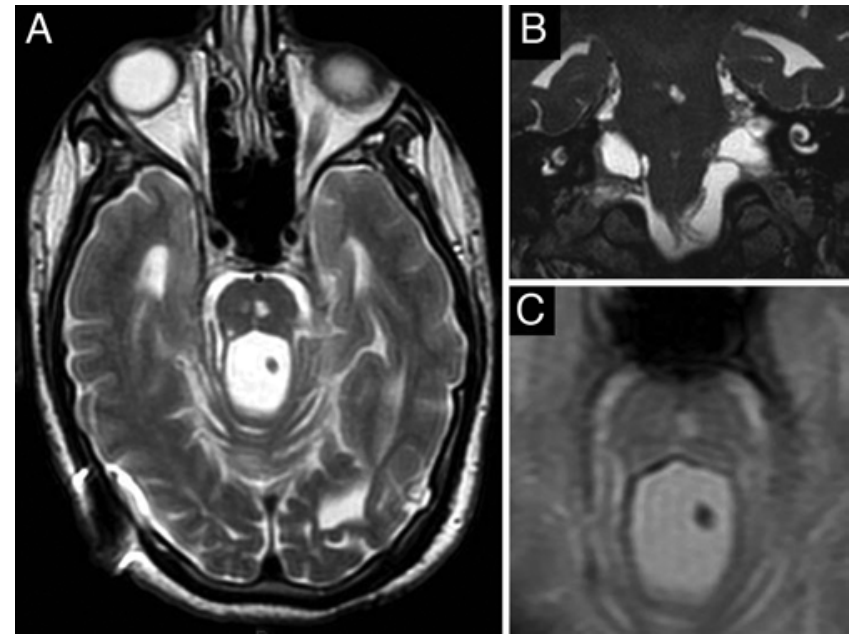

FIG. 3. Axial (A) and coronal (B) T2-weighted MRI sequences obtained 1 month after DTH occurred, showing small lacunae in the pons. Gradient echo axial image $(C)$ ruling out the hemorrhagic nature of these foci.

According to our hydrodynamic hypothesis, the infratentorial shunt brought about a negative pressure gradient between the supratentorial compartment and the fourth ventricle, with a progressive dilation of the supratentorial ventricles that finally resulted in DTH. Interestingly, this picture was initially misdiagnosed as a malfunction of the supratentorial shunt. However, the supratentorial ventricles remained large, in spite of very low-pressure external drainage $\left(<5 \mathrm{~cm} \mathrm{H}_{2} \mathrm{O}\right)$, while the fourth ventricle shunt was left in place. Only the exteriorization of this shunt and a higher drainage pressure of the fourth ventricle solved this problem, further corroborating our hydrodynamic hypothesis. It is worth noting that supratentorial ventricles dilated in spite of the presence of a working shunt or external CSF drainage. Although we would expect a radiological picture of overdrainage with slit ventricles and extracranial fluid collections, this did not occur, probably due to the patient's perinatal history of hemorrhage and infection resulting in the scarification of subarachnoid convexity spaces and the chronic CSF drainage with subsequent fibrotic subdural space.

Interestingly, a similar hydrodynamic mechanism has been described in uncal herniation secondary to posterior fossa shunting. ${ }^{26}$ Another condition that eventually shares the same pathogenesis is paradoxical herniation, which is a peculiar form of DTH resulting from intracranial hypotension secondary to lumbar CSF drainage in the absence of protective bone, as occurs during a craniotomy or after decompressive craniectomy. ${ }^{16,18}$ Differently from our case, paradoxical herniation occurs in an unpartitioned and contiguous compartment.

Although the mechanism of DTH due to a CSF pressure gradient without mass lesion needs to be further elucidated, the risk of its occurrence should be kept in mind. To minimize this risk, the endoscopic treatment options aiming at reestablishing the communication between the trapped fourth ventricle and the supratentorial ventricles should be preferred to other strategies based on multiple shunting procedures. 
The mechanism of brainstem sufferance complicating DTH in the present case deserves further discussion. In fact, DTH, regardless of its etiology, displaces the upper brainstem caudally and causes an abrupt side-to-side compression at the anteroposterior axis of the brainstem. ${ }^{2}$ This results in secondary brainstem hemorrhages with a peculiar distribution in the rostral midbrain and in the ventral portion of the pons, presenting in a delayed fashion, that are termed Duret hemorrhages. ${ }^{1,8,17}$ The pathogenesis of Duret hemorrhages has been hypothesized to consist of 2 mechanisms, stretching and disruption of small central perforating arteries or compression of small rostral draining veins, both ensuing from the abrupt descent of the brainstem against the basilar artery, which is relatively immobilized by the circle of Willis. ${ }^{23}$ In the present case, the posthemorrhagic and postinfectious etiology of the hydrocephalus may have contributed to the arachnoidal scarring and relative immobilization of the basilar artery. However, the pathogenetic mechanism based on a minimal pressure gradient between the supra- and infratentorial compartment, and in particular the absence of an increased pressure in the supratentorial compartment, eventually led to a more gradual and subtle distortion of the brainstem and its vessels. According to this hypothesis, MRI revealed ischemic foci in the brainstem, without any signs of hemorrhage.

As a consequence, the absence of hemorrhagic infarction of the brainstem lesions may partly account for the good outcome in the present case. In fact, Duret hemorrhages are generally burdened by a very high incidence of mortality or persistent vegetative state. The poor outcome of most of the cases described in the literature may be partly related to the underlying traumatic etiology, with rapidly evolving mass lesions. Indeed, a nontraumatic etiology, usually consistent with paradoxical herniation after lumbar puncture or rapid correction of hyponatremia, is extremely rare. ${ }^{13,16,18}$ Good neurological outcome after Duret hemorrhages is seldom reported in adults and is usually related to timely evacuation of the mass lesion that determines DTH. Recently the contribution of brain plasticity has been also invoked to explain the good recovery of 2 pediatric cases with traumatic brain injury. ${ }^{1}$

In conclusion, DTH may represent an unusual complication in the management of trapped fourth ventricle. Endoscopic aqueductoplasty, whenever feasible, should be preferred to multiple shunting procedures to avoid the risk of differential pressure between the supratentorial and infratentorial compartment, which is the pathogenetic mechanism of this complication. DTH may cause brainstem sufferance, usually associated with the radiological picture of Duret hemorrhages. Although brainstem sufferance is usually associated with a dismal prognosis, in this setting it may show a more favorable outcome because of the slow and insidious pathogenetic mechanism without abrupt increase of supratentorial pressure.

\section{References}

1. Beier AD, Dirks PB: Pediatric brainstem hemorrhages after traumatic brain injury. J Neurosurg Pediatr 14:421-424, 2014

2. Chi NF, Wang SJ, Lirng JF, Fuh JL: Transtentorial herniation with cerebral infarction and Duret haemorrhage in a patient with spontaneous intracranial hypotension. Cephalalgia 27:279-282, 2007

3. Cinalli G, Spennato P, Savarese L, Ruggiero C, Aliberti F, Cuomo L, et al: Endoscopic aqueductoplasty and placement of a stent in the cerebral aqueduct in the management of isolated fourth ventricle in children. J Neurosurg 104 (1 Suppl):21-27, 2006

4. Dollo C, Kanner A, Siomin V, Ben-Sira L, Sivan J, Constantini S: Outlet fenestration for isolated fourth ventricle with and without an internal shunt. Childs Nerv Syst 17:483-486, 2001

5. Feldmann E, Gandy SE, Becker R, Zimmerman R, Thaler HT, Posner JB, et al: MRI demonstrates descending transtentorial herniation. Neurology 38:697-701, 1988

6. Fritsch MJ, Kienke S, Manwaring KH, Mehdorn HM: Endoscopic aqueductoplasty and interventriculostomy for the treatment of isolated fourth ventricle in children. Neurosurgery 55:372-377, discussion 377-379, 2004

7. Fritsch MJ, Kienke S, Mehdorn HM: Endoscopic aqueductoplasty: stent or not to stent? Childs Nerv Syst 20:137-142, 2004

8. Fujimoto Y, Aguiar PH, Freitas AB, de Andrade AF, Marino Júnior R: Recovery from Duret hemorrhage: a rare complication after craniotomy-case report. Neurol Med Chir (Tokyo) 40:508-510, 2000

9. Gallo P, Szathmari A, Simon E, Ricci-Franchi AC, Rousselle C, Hermier M, et al: The endoscopic trans-fourth ventricle aqueductoplasty and stent placement for the treatment of trapped fourth ventricle: long-term results in a series of 18 consecutive patients. Neurol India 60:271-277, 2012

10. Harter DH: Management strategies for treatment of the trapped fourth ventricle. Childs Nerv Syst 20:710-716, 2004

11. Ishizaka $S$, Shimizu $T$, Ryu N: Dramatic recovery after severe descending transtentorial herniation-induced Duret haemorrhage: a case report and review of literature. Brain Inj 28:374-377, 2014

12. James HE: Spectrum of the syndrome of the isolated fourth ventricle in posthemorrhagic hydrocephalus of the premature infant. Pediatr Neurosurg 16:305-308, 1990-1991

13. Kamijo Y, Soma K, Kishita R, Hamanaka S: Duret hemorrhage is not always suggestive of poor prognosis: a case of acute severe hyponatremia. Am J Emerg Med 23:908-910, 2005

14. Marupaka SK, Sood B: Atypical Duret haemorrhages seen on computed tomography. Emerg Med Australas 20:180182,2008

15. Montes JL, Clarke DB, Farmer JP: Stereotactic transtentorial hiatus ventriculoperitoneal shunting for the sequestered fourth ventricle. Technical note. J Neurosurg 80:759-761, 1994

16. Oyelese AA, Steinberg GK, Huhn SL, Wijman CA: Paradoxical cerebral herniation secondary to lumbar puncture after decompressive craniectomy for a large space-occupying hemispheric stroke: case report. Neurosurgery 57:E594, 2005

17. Parizel PM, Makkat S, Jorens PG, Ozsarlak O, Cras P, Van Goethem JW, et al: Brainstem hemorrhage in descending transtentorial herniation (Duret hemorrhage). Intensive Care Med 28:85-88, 2002

18. Samadani U, Huang JH, Baranov D, Zager EL, Grady MS: Intracranial hypotension after intraoperative lumbar cerebrospinal fluid drainage. Neurosurgery 52:148-152, 2003

19. Sansone JM, Iskandar BJ: Endoscopic cerebral aqueductoplasty: a trans-fourth ventricle approach. J Neurosurg 103 (5 Suppl):388-392, 2005

20. Schulz M, Goelz L, Spors B, Haberl H, Thomale UW: Endoscopic treatment of isolated fourth ventricle: clinical and radiological outcome. Neurosurgery 70:847-859, 2012 
21. Sharma RR, Pawar SJ, Devadas RV, Dev EJ: CT stereotaxy guided lateral trans-cerebellar programmable fourth ventriculo-peritoneal shunting for symptomatic trapped fourth ventricle. Clin Neurol Neurosurg 103:143-146, 2001

22. Shin M, Morita A, Asano S, Ueki K, Kirino T: Neuroendoscopic aqueductal stent placement procedure for isolated fourth ventricle after ventricular shunt placement. Case report. J Neurosurg 92:1036-1039, 2000

23. Stiver SI, Gean AD, Manley GT: Survival with good outcome after cerebral herniation and Duret hemorrhage caused by traumatic brain injury. J Neurosurg 110:1242-1246, 2009

24. Teo C, Burson T, Misra S: Endoscopic treatment of the trapped fourth ventricle. Neurosurgery 44:1257-1262, 1999

25. Torres-Corzo J, Rodriguez-Della Vecchia R, Rangel-Castilla $\mathrm{L}$ : Trapped fourth ventricle treated with shunt placement in the fourth ventricle by direct visualization with flexible neuroendoscope. Minim Invasive Neurosurg 47:86-89, 2004

26. Udayakumaran S, Ben Sira L, Constantini S: Chronic uncal herniation secondary to posterior fossa shunting: case report and literature review. Childs Nerv Syst 26:267-271, 2010

27. Udayakumaran S, Biyani N, Rosenbaum DP, Ben-Sira L, Constantini S, Beni-Adani L: Posterior fossa craniotomy for trapped fourth ventricle in shunt-treated hydrocephalic children: long-term outcome. J Neurosurg Pediatr 7:52-63, 2011

28. Udayakumaran S, Panikar D: Postulating the concept of compensated trapped fourth ventricle: a case-based demonstration with long-term clinicoradiological follow-up. Childs Nerv Syst 28:661-664, 2012

\section{Author Contributions}

Conception and design: Frassanito. Acquisition of data: Di Bonaventura. Analysis and interpretation of data: Markogiannakis. Drafting the article: Frassanito, Markogiannakis, Di Bonaventura. Critically revising the article: Frassanito, Caldarelli. Reviewed submitted version of manuscript: Massimi, Tamburrini. Approved the final version of the manuscript on behalf of all authors: Frassanito. Study supervision: Caldarelli.

\section{Correspondence}

Paolo Frassanito, Pediatric Neurosurgery, Catholic University Medical School, Largo Agostino Gemelli, 8, Rome 00168, Italy. email: paolo.frassanito@gmail.com. 